\title{
A SNP of HD-ZIP I transcription factor leads to distortion of trichome morphology in cucumber (Cucumis sativus L.)
}

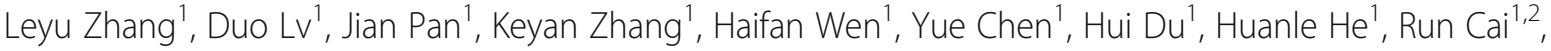 \\ Junsong Pan ${ }^{1 *}$ and Gang Wang ${ }^{1 *}$
}

\begin{abstract}
Background: Trichomes are excellent model systems for the analysis of cell differentiation and play essential roles in plant protection. From cucumber inbred line 'WD1', we identified an EMS-induced trichome abnormally developing mutant, nps, which exhibited smaller, denser and no pyramid-shaped head trichomes.

Results: Using $F_{2}$ and $B_{1}$ populations constructed from a cross between $n p s$ and '9930', the genetic analysis showed that the nps trait is controlled by a single recessive nuclear gene. We identified CsNps by map-based cloning with 576 individuals of the $F_{2}$ population generated from the cross of nps and inbred line ' 9930 '. The CsNps was located at a 13.4-kb genomic region on chromosome 3, which region contains three predicted genes. Sequence analysis showed that only one single nucleotide mutation $(C \rightarrow T)$ between 9930 and nps was found in the second exon of Csa3G748220, a plant-specific class I HD-Zip gene. The result of allelism test also indicated that nps is a novel allelic mutant of Mict (Micro-trichome). Thus, nps was renamed mict-L130F. By comparing the transcriptome of mict-L130F vs WD1 and 06-2 (mict) vs 06-1 (wildtype, near-isogenic line of 06-2), several potential target genes that may be related to trichome development were identified.
\end{abstract}

Conclusions: Our results demonstrate that Mict-L130F is involved in the morphogenesis of trichomes. Map-based cloning of the Mict-L130F gene could promote the study of trichome development in cucumber.

Keywords: Cucumber, Trichome, Map-based cloning, Transcriptome

\section{Introduction}

Trichomes are large specialized epidermal cells distributed on aerial parts [1-3]. In different species, the trichome can be classified as unicellular or multicellular, glandular or non-glandular, and branched or unbranched [4]. Trichomes play important roles in protecting plants from biotic and abiotic stresses, such as insect predation, excess transpiration and UV light $[5,6]$. The development of unicellular and

\footnotetext{
* Correspondence: jspan71@sjtu.edu.cn; wg770801@sjtu.edu.cn 'School of Agriculture and Biology, Shanghai Jiao Tong University, Shanghai 200240, China

Full list of author information is available at the end of the article
}

multicellular trichomes may be controlled by different pathways.

In Arabidopsis, as giant single epidermal cells, trichomes have become an excellent model system to study cell differentiation and development because it is easy to analyze them at the genetic, genomic and cell biology levels. Different trichome patterning methods depend on complicated gene expression and intercellular signal transduction [4]. In Arabidopsis leaf epidermal cells, the MYB-bHLH-WDR (MBW) complex is formed by the R2R3 type MYB transcript factor GLABRA 1 (GL1), bHLH transcript factors GLABRA 3/ENHANCER OF GLABRA 3 (GL3/EGL3), and WD40 repeat protein

(c) The Author(s). 2021 Open Access This article is licensed under a Creative Commons Attribution 4.0 International License, which permits use, sharing, adaptation, distribution and reproduction in any medium or format, as long as you give appropriate credit to the original author(s) and the source, provide a link to the Creative Commons licence, and indicate if changes were made. The images or other third party material in this article are included in the article's Creative Commons licence, unless indicated otherwise in a credit line to the material. If material is not included in the article's Creative Commons licence and your intended use is not permitted by statutory regulation or exceeds the permitted use, you will need to obtain permission directly from the copyright holder. To view a copy of this licence, visit http://creativecommons.org/licenses/by/4.0/ The Creative Commons Public Domain Dedication waiver (http://creativecommons.org/publicdomain/zero/1.0/) applies to the data made available in this article, unless otherwise stated in a credit line to the data. 
TRANSPARENT TESTA GLABRA 1 (TTG1). GLABRA 2 (GL2) can be activated by the MBW complex and then turn on trichome initiation [7-13]. In mature trichomes, GL2 is highly expressed and is necessary for trichome initiation and maintenance during cell differentiation [14]. The R3 type MYB transcription factors are also activated by the MBW complex CAPRICE (CPC), ENHA NCER OF TRY AND CPC 1 (ETC1), ETC2, and ETC3 which all belong to the transcription factors and act as negative regulators in trichome development [4, 15-18]. The R3 type MYB transcription factors can move to neighboring cells and interact with GL3 and TTG1, forming the different R3 type MBW complexes. This complex inhibits trichome differentiation in neighboring cells as it cannot activate GL2 expression [18, 19].

Cucumber (Cucumis sativus L.) is one of the most important commercial vegetables worldwide and has become a model plant for studying the formation and development of multicellular trichomes. Trichomes are widely distributed on different organs, such as leaves, stems, branches, flowers, tendrils, and fruits, in cucumber. The trichomes on the fruits are commonly called "fruit spines". Fruit spines directly influence fruit appearance and perceived quality. In contrast to Arabidopsis, a limited number of cucumber genes related to the formation and development of trichomes have been identified. Among them, tril (trichome-less) and csgl3 (glabrous3) are alleles with different mutation forms of the same gene (Csa6G514870). In tril and $\operatorname{csg} l 3$, a glabrous character is observed on stems, leaves, tendrils, receptacles and ovaries, and there were no spines or tumors on the fruit surface [2023]. The micro-trichome (mict), tiny branched hair (tbh), and glabrous 1 (csgll) alleles were identified as the result of the same $2649 \mathrm{bp}$ fragment deletion in the gene Csa3G748220. These mutants have no noticeable trichomes on the surface of stems, leaves, tendrils, and ovaries [24-26]. The no warty phenotype of tuberculate fruit $(t u)$ was due to complete deletion of the $4888 \mathrm{bp}$ region that included the promoter and CDS region of Csa5G577350 (Tu) [27].

In this study, a novel no pyramid-shaped head trichome (nps) mutant was identified in an ethyl methane sulfonate (EMS)-mutagenized WD1 (North China type) cucumber population. We isolated the nps gene using map-based cloning and found that nps is the allele of mict with a single nucleotide substitution. Thus, we renamed the gene Mict-L130F. In addition, transcriptome analysis was conducted to identify the genes involved in the formation and development of malformed trichomes/spines in cucumber. This work enriches the phenotype of trichomes and will promote the exploration of molecular mechanisms that regulate cucumber trichome development.

\section{Materials and methods}

Plant materials and phenotypic data collection

The no pyramid-shaped head trichome (nps) mutant was identified from an $\mathrm{M}_{2}$ family derived from an EMSmutagenized cucumber WD1 (WT, North China type) population. Mutagenized plants of the first mutant generation $\left(M_{1}\right)$ were self-pollinated for two generations to make the mutated gene homozygous, and then $F_{2}$ and $\mathrm{BC}_{1}$ populations were produced with the nps (female) and 9930 (North China type) (male) as the parents. $\mathrm{Cu}$ cumber line $06-2$ is the spontaneous mict mutant from the North China inbred line 06-1(wild type), which are near-isogenic lines [26]. All of the plants were grown under natural sunlight in a greenhouse at Shanghai Jiao Tong University.

The leaf phenotype of each plant was determined by visual inspection when leaves were fully expanded. The chi-square goodness of fit test was performed on phenotypic data to verify deviations from the expected 3:1 segregation in the $F_{2}$ population or 1:1 segregation in the $\mathrm{BC}_{1}$ population.

\section{Scanning electron microscopy (SEM) analysis}

Juvenile leaf ( $3 \mathrm{~cm}$ in length) and fruit (4 $\mathrm{cm}$ in length) samples of WT and nps were harvested and fixed in formaldehyde acetic acid-ethanol (FAA) which contained $50 \%(\mathrm{v} / \mathrm{v})$ ethanol, $5 \%(\mathrm{v} / \mathrm{v})$ acetic acid, and $3.7 \%(\mathrm{v} / \mathrm{v})$ formaldehyde at $4{ }^{\circ} \mathrm{C}$ for $24 \mathrm{~h}$, dehydrated through a graded ethanol series $(50,60,70,85,90,95$, and $100 \%)$, critical-point dried in a Leica EM CPD030 desiccator (Leica Microsystems, Wetzlar, Germany), coated with gold-palladium in a Hitachi E-1045 ion sputter and carbon coating unit (Hitachi, Tokyo, Japan), and then observed through a JSM-6360LV scanning electron microscope (JEOL, Peabody, MA, USA) [28].

\section{Molecular marker development and map-based cloning} Deletion-insertion (InDel) and single nucleotide polymorphism (SNP) markers were developed based on genome resequencing. The genome of WT was resequenced on the Illumina HiSeq 2000 platform (Biomarker Technologies, Beijing, China). With a 30 -fold sequencing depth, all of the clean reads were mapped to the '9930' genome sequence (http://cucurbitgenomics.org/ organism/2, version 2i). InDel and SNP sites between WD1 and 9930 were detected with the Geneious software package. Only fragments with a more than $3 \mathrm{bp}$ insertion or deletion were used to develop InDel markers. For SNP genotyping, approximately $800 \mathrm{bp}$ fragments, including the SNP site in the middle of the fragment, were amplified and sequenced. The primers were designed with Primer Premier 5.0. All SSR primers used in the study were kindly provided by the group of professor 
Sanwen Huang (Chinese Academy of Agricultural Sciences, Beijing, China).

The bulked segregant analysis (BSA) method [29] was used for screening polymorphic markers and mapping the nps locus. Ten individuals were randomly selected from the no pyramid-shaped head trichomes and wild type phenotype plants from the $F_{2}$ population to create two pools ( $\mathrm{M}$ and W DNA pools). With the 72 individuals of the $F_{2}$ population for initial mapping, the nps was located on chromosome 3 (Chr3). An additional larger $\mathrm{F}_{2}$ population with 504 individuals generated from 9930 and $n s p$ was then used for fine mapping of the nps locus with SNP markers and InDel markers [30-32]. Genetic maps were drawn with Join-Map 4.0. Information on all newly developed markers is provided in Table S1.

\section{DNA extraction and molecular marker analysis}

Genomic DNA was extracted from young leaves using the CTAB method [33]. For the SSR and InDel markers, PCRs were carried out using a $10 \mu \mathrm{l}$ volume containing $40 \mathrm{ng}$ genomic DNA, $0.5 \mu \mathrm{M}$ each primer, $200 \mu \mathrm{M}$ dNTPs, $1 \times$ reaction buffer, and $0.5 \mathrm{U}$ Taq DNA polymerase (Takara Bio Inc., Beijing, China). PCR amplification was performed on a PCR thermocycle instrument (Applied Biosystems, Foster, USA) using the following PCR program: $94{ }^{\circ} \mathrm{C}$ for $5 \mathrm{~min}$; 35 cycles of $94^{\circ} \mathrm{C}$ for 30 s, $50-60{ }^{\circ} \mathrm{C}$ for $30 \mathrm{~s}, 72{ }^{\circ} \mathrm{C}$ for $30 \mathrm{~s}$; and a final $72{ }^{\circ} \mathrm{C}$ for $5 \mathrm{~min}$. Products were separated on an $8 \%$ polyacrylamide gel by electrophoresis. After electrophoresis at $220 \mathrm{~V}$ for $1.5 \mathrm{~h}$, the gel was separated from the plates and stained in $0.2 \% \mathrm{AgNO}_{3}$ solution (Shanghai Shi Yi chemicals Reagent, Shanghai, China). Finally, the stained gel was transferred into the developing solution (1.5\% sodium hydroxide and $0.4 \%$ formaldehyde) to reveal the silverstained DNA bands. For SNP markers, the PCR reaction and PCR amplification were the same as for the SSR markers. The reaction samples were $20 \mu \mathrm{l}$ in volume, and the products were analyzed by sequencing (Sangon Biotech, Shanghai, China) [34].

\section{Sample collection and qRT-PCR}

The apical leaves (three independent biological replicates) were collected from WT and nps. Total RNA was extracted using an OminiPlant RNA Kit (DNase I) (CWBIO, Nanjing, China). First-strand cDNA was prepared according to the HiFiScript cDNA Synthesis Kit (CWBIO, Nanjing, China) protocol. Quantitative PCR (qPCR) was conducted using FastStart Universal SYBR Green Master Mix (ROX) (Roche) with a CFX96 Touch ${ }^{\mathrm{Tx}}$ Real-Time PCR System. The cucumber CsActin gene was selected as an internal control [35]. Three biological replicates were used per gene. Each qRT-PCR experiment was performed with three technical replicates. The gene-specific primers are listed in Table S1.

\section{Materials for RNA-seq}

Total RNA was extracted from the apical leaves. RNASeq for comparative transcriptomic analyses of the two phenotypes was performed with three biological replicates. Library construction and sequencing were performed using a BGISEQ-500 by Beijing Genomic Institution (BGI, China). The genomic DNA was removed with two digestions using amplification grade DNase I (Epigenetics, United States). The RNA was sheared and reverse transcribed using random primers to obtain cDNA, which was used for library construction. The library quality was determined using a Bioanalyzer 2100 (Agilent), and then the library was used for sequencing using the sequencing platform BGISEQ-500 (BGI, China) [36]. All the generated raw sequencing reads were filtered to remove reads with adaptors and reads in which unknown bases were greater than $10 \%$ of low-quality reads. The clean reads were obtained and saved in FASTQ format. We used Bowtie2 [37] to map the clean reads to the reference genome, (Cucumber ChineseLong_v2 1, [38]). The read counts were summarized, and the Fragments Per Kilobase of exon per Million fragments mapped (FPKM) was calculated for each annotation on the reference sequence. The NOISeq method [39] was used to screen for DEGs between the groups. An expression trend analysis was performed using OmicShare tools (http://www.omicshare.com/ tools). The clean data have been uploaded to the $\mathrm{Na}$ tional Center for Biotechnology Information (NCBI) (Project ID: PRJNA706516, PRJNA706464, PRJNA706461, PRJNA706463,PRJNA706462, and PRJNA706166).

\section{Yeast one-hybrid assay}

For the yeast one-hybrid assay, the Mict-L130F open reading frames (ORFs) were amplified from $n p s$ genomic DNA and then cloned into the $\mathrm{pB} 42 \mathrm{AD}$ vector. The $2 \mathrm{~kb}$ promoter of CsTT4, CsFLS1, CsCER26 and CsMYB36 from WD were inserted into the vector. pB42AD-MictL130F and placZ-CsTT4Pro, placZ-CsFLS1Pro, placZCsCER26Pro, or placZ-CsMYB36Pro were cotransformed into the yeast strain EGY48a. The empty vectors were used as negative controls [40]. Primers are listed in Table S1.

\section{Results}

\section{Phenotypic characterization}

Both the nps and WT are covered by trichomes on male flowers, tendrils, fruits and leaves. Compared with the WT trichomes, the nps trichomes appeared to be short and tender on leaves, female flowers, and tendrils or spines on fruit (Fig.1 a-d). 9930, which was used for producing the $F_{2}$ population for map-based cloning, shows a similar phenotype to WT in leaves, stems, and other 

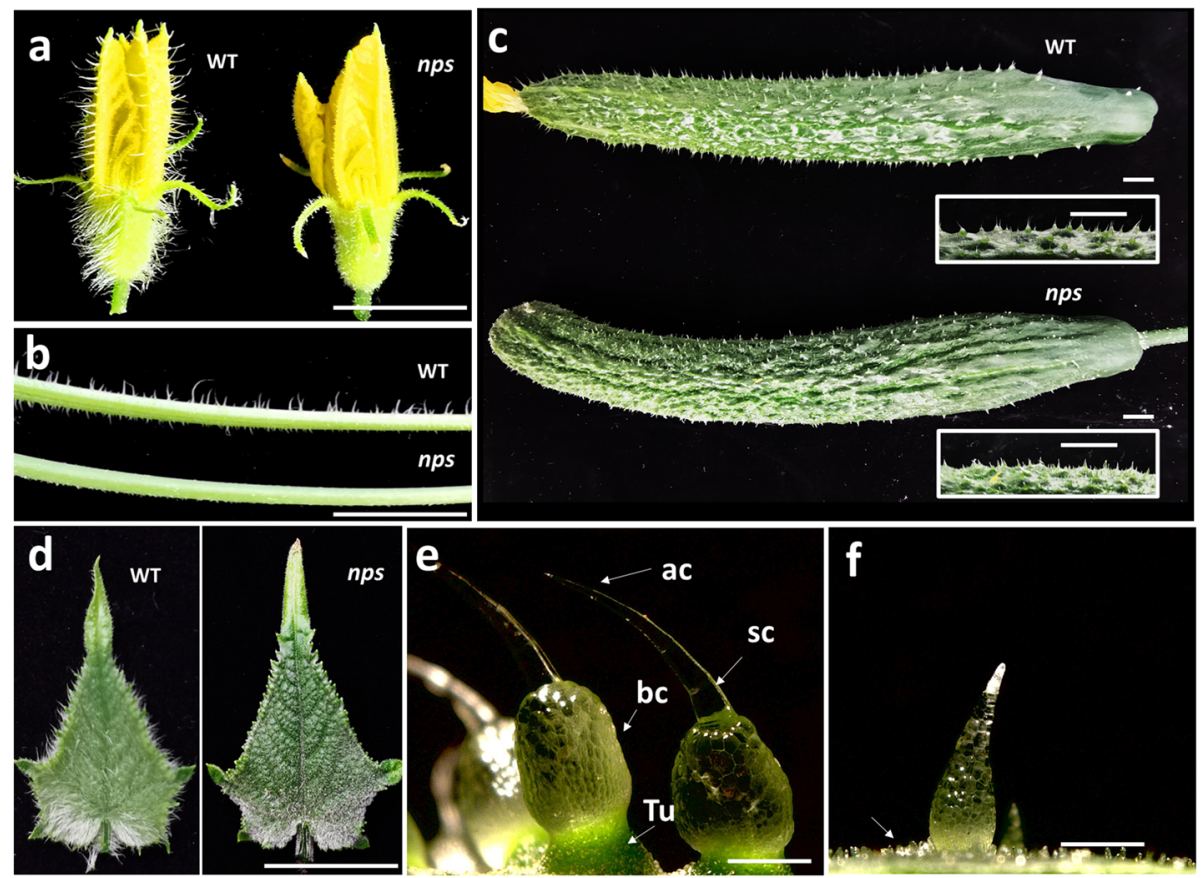

Fig. 1 Phenotype of nps and WT plants. (a-d) Show male flower, tendril, fruit and leaf of WT and nps. e Fruit spines of WT; arrows indicate tubercule (Tu), base cell (bc), stalk cell (sc), and apical cell (ac). f Fruit spines of nps; arrow indicates bumps. Bars: $1 \mathrm{~cm}(\mathrm{a}-\mathrm{d}), 100 \mu \mathrm{m}$ (e-f)

organs. We examined the cucumber fruit spines with an optical microscope. Obviously, spines on the WT fruits are much larger than those on the nps fruits. In addition, the structures of the fruit spines are different between WT and nps. In the WT, the spines consist of pyramidshaped apical cells, slender and elongated stalk cells and enlarged base cells. The tuberculate connects the spines to the epidermis (Fig. 1e). In the nps, the fruit spines are conical in shape but without sharp tips. Many small and dense bumps are visible around the spines (Fig. 1f).

To characterize the morphology of cucumber trichomes in detail, we observed leaf trichomes and fruit spines with SEM. In the WT, the majority of trichomes are non-glandular and consist of pyramid-shaped apical cells, multicellular stalks with two to four elongated cylindrical-shaped cells and pie-shaped base cells. The minority were glandular trichomes and had a miniround shape (Fig. 2a). In the nps, there were no enlarged basal cells in the trichome, and flat cylinder stalk cells connected the apical cell to the leaf surface (Fig. 2b). There are two types (type I and type II) of trichomes in the mutant. The main difference between type I and type II is the shape of apical cells. The apical cell shape of type I (Fig. 2c) is round; however, the shape of type II (Fig. 2d) is papillar-shaped.

In the WT, the fruit spines were distinct with clear fruit tubercules and the base of the spine was made of hundreds of spherical-shaped cells whose sizes were smaller than those of the stalk cells. The stalk consists of three to seven cylindrical-shaped cells plus a pyramid-shaped apical cell (Fig. 2e). In the nps, there are three types (type I, type II, and type III) of spines, which are crowded together (Fig. 2f). Type I is branchless and cone-shaped without sharp apical cells and fruit tubercules. The whole spines consist of spherical-shaped cells similar to the base of the wildtype spine with three to four flat cells on the top. Type II has branches, and the apical cell is spherical. The size of type II is smaller than that of type I because type ii has fewer cells. Type III is the smallest, with a greatly reduced number of cells and it is difficult to separate the base and stalk; however, the apical cell is also spherical (Fig. 2g).

To further detect the development of trichomes, we observed changes in the leaf primordium during germination between the WT and mutant through optical microscopy and SEM. Based on the morphology, the initiative development of trichomes is divided into five stages (Fig. 3). In stage I, no trichomes appear on the leaf primordium. In Stage II, several bulges emerge on the leaf surface. In Stage III, a mass of bulges form, and their shapes are transformed into cone-like structures. In Stage IV, multicellular trichomes begin to develop. In Stage V, the morphology and density of the trichomes are basically formed and the trichomes further elongate during the development of leaves. The greatest difference between WT and nps occurs from Stages III to V. From Stage III, pyramid-shaped apical cells fail to 


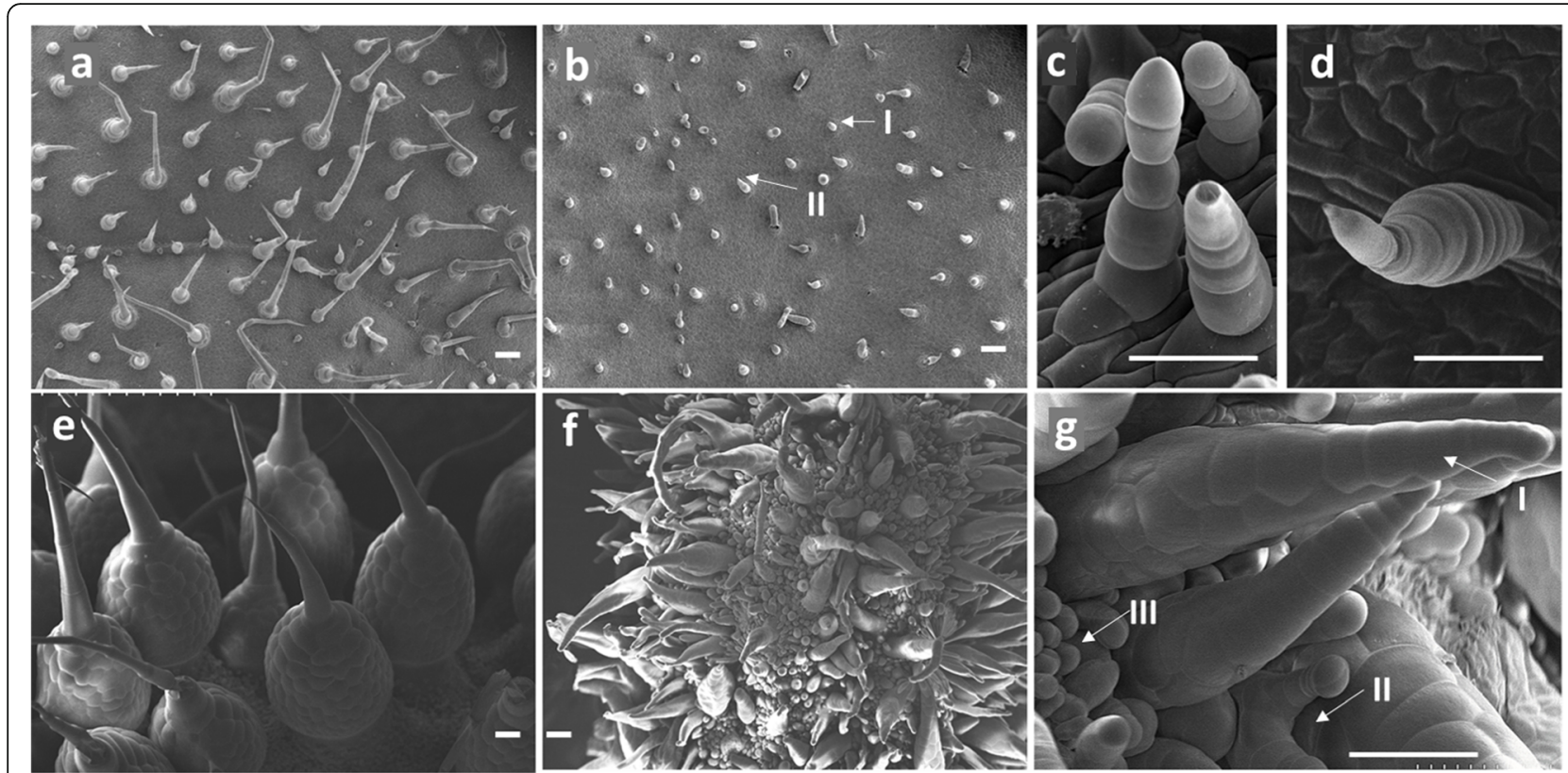

Fig. 2 SEM images of trichomes and fruit spines between the WT and nps. a trichomes on WT leaf. b-d trichomes on nps leaf. e spines on WT fruit. (f) and $\mathbf{g}$ spines on $n p s$ fruit. Arrows indicate type I, type II and type III. Scale bars: $50 \mu \mathrm{m}(\mathrm{a}-\mathrm{d}), 100 \mu \mathrm{m}(\mathbf{e}-\mathbf{g})$

develop in the nps. These results indicated that the nps functioned in the morphogenesis of trichomes.

\section{The $n p s$ locus is controlled by a recessive nuclear gene in} cucumber

The trichomes/fruit spines of all the $\mathrm{F}_{1}$ plants produced from the cross of nps and 9930 were consistent with those of 9930. No significant phenotypic difference was found between homozygous dominant (Nps/Nps) and heterozygous (Nps/nps) plants. Of the $178 n p s \times 9930 \mathrm{~F}_{2}$ plants, 132 and 46 plants exhibited 9930 and mutant phonotypes, respectively. Goodness-of-fit $X^{2}$ tests indicated that the segregation ratios in the $F_{2}\left(X^{2}=\right.$ $\left.0.029<\chi_{0.05,1}^{2}=3.84\right)$ populations were consistent with the expected 3:1 segregation ratio. Among 186 (nps $\times$ 9930) $\times n p s \quad \mathrm{BC}_{1}$ individuals, 87 and 99 plants exhibited 9930 and mutant phonotypes, respectively, conforming to the $1: 1$ segregation ratio $\left(\chi^{2}=0.65<\chi_{0.05,1}^{2}=3.84\right)$. These results indicated that both the $\mathrm{F}_{2}$ and $\mathrm{BC}_{1}$ populations conformed to Mendel segregation which confirmed that $n p s$ was conferred by a single recessive nuclear gene in cucumber.

\section{Primary mapping of the nps locus}

Genetic mapping of the nps locus was performed using the BSA approach. Polymorphic SSR markers between $n p s$ and 9930 were identified. Based on the resequencing genomic data, 73 polymorphic InDel markers between nps and 9930 were developed. All 56 polymorphic SSR markers and 73 polymorphic InDel markers between nps and 9930 were used to analyze the W and M DNA pools. Among these markers, five markers (SSR04724, Indel3-14, Indel3-27, SSR11397, SSR13974) were polymorphic between the $\mathrm{W}$ and M DNA pools. The five markers were located on chromosome 3 in cucumber. Genetic linkage analysis using 72 individuals of the $F_{2}$ population showed that nps was linked with these five markers (Fig. 4a). The results indicated that nps was located on chromosome 3, within an approximately 6.7 cM interval between Indel3-14 and Indel3-27 (Fig. 4a). To narrow down the target region, three new polymorphic InDel markers (Indel3-42, Indel3-56, Indel370) between Indel3-14 and Indel3-27 were developed based on the genomic sequence (Fig. 4b). The nps locus was mapped between the markers Indel3-56 and Indel $3-70$ on chromosome 3 by genotyping $504 \mathrm{~F}_{2}$ individuals (Fig. 4b). A total of 21 recombinants were identified between Indel 3-56 and Indel 3-70 by genotyping 504 individuals.

\section{Fine mapping of the nps locus}

According to the resequencing genome data, there were no more polymorphic InDel markers between Indel3-56 and Indel3-70. Therefore, SNP markers were developed for fine mapping. Five SNP markers were developed and these SNP markers showed polymorphisms between nps and 9930. The 21 recombinants between Indel3-56 and Indel3-70 were used for fine mapping with the polymorphic SNP markers. Finally, the nps locus was mapped to a region between SNP3 and SNP4 markers, encompassing a physical distance of $13.4 \mathrm{~kb}$ (Fig. 4c). 


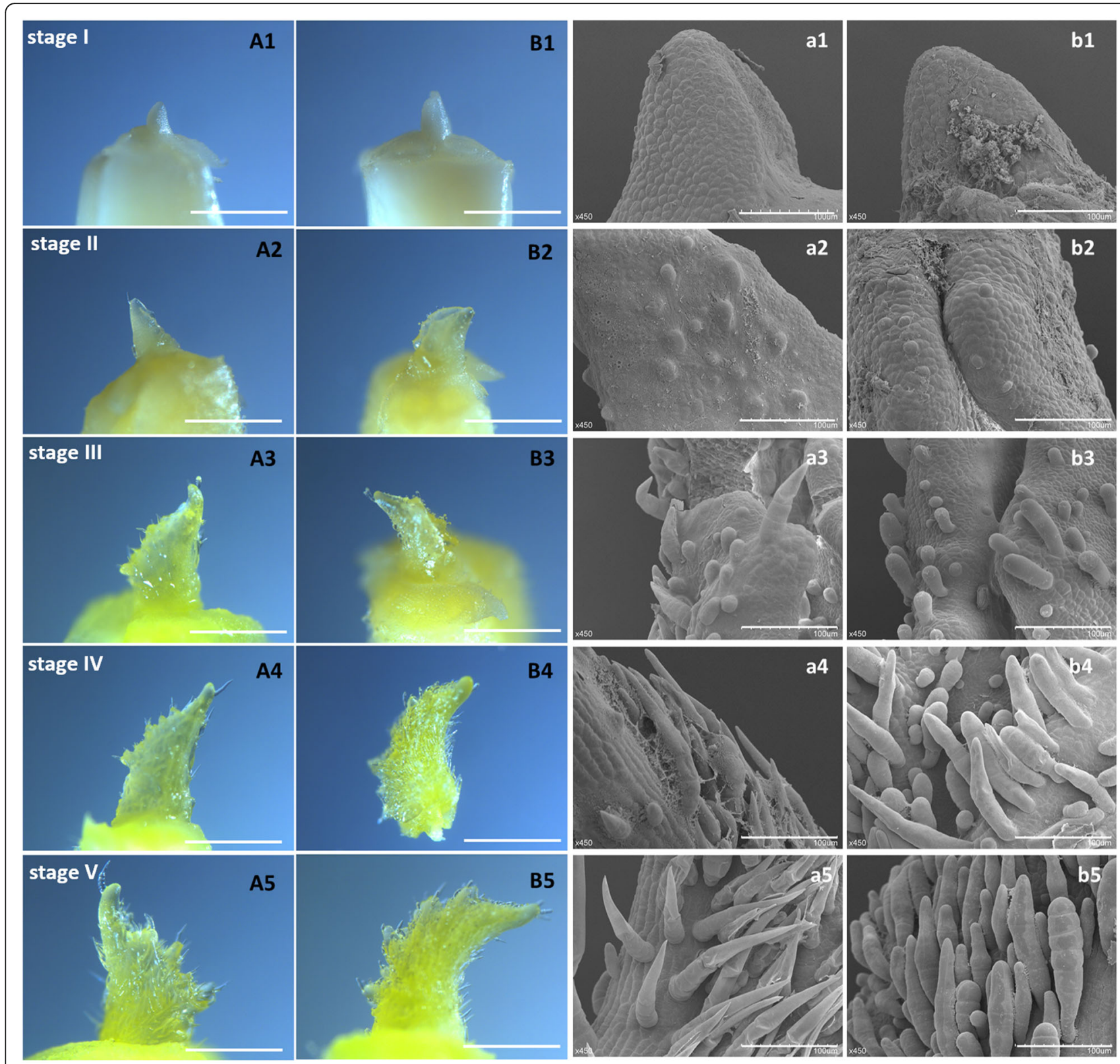

Fig. 3 Five stages of trichomes morphological development. (A1-A5) and (a1-a5) are leaves of WT. (B1-B5) and (b1-b5) are leaves of nps. Bars: 1 cm (A1-A5, B1-B5), $100 \mu m(a 1-a 5, ~ b 1-b 5)$

\section{Candidate gene identification}

In the 13.4-kb genomic region, three putative genes were identified in the "9930" genome database (http:// cucurbit-genomics.org/organism/2). The information and predicted functions of the three genes are presented in Table 1. After analyzing the coding sequences and the protomer regions of the three genes of 9930 and nps, only one nonsynonymous single nucleotide mutation was identified in gene Csa3G748220, whereas no difference was found in the other two genes (Fig. 4d). In addition, the single nucleotide mutation of the candidate gene only existed in the nps mutant but not in the other
10 cucumber lines of the natural populations (File S1). The data suggested that this SNP may be the causal SNP for the trichome phenotype. We also examined the linkage relationship of this SNP with the nps locus. A dCAPS marker (dcaps-M) developed based on this SNP (primer information in Table S1) was used to genotype 504 nps $\times 9930 \mathrm{~F}_{2}$ plants. The results showed that dcaps-M co-segregated with the nps locus in this population (Figure S1). Therefore, we concluded that Csa3G748220, a class I Homeodomain-leucine zipper (HD-ZIP) gene, carrying the SNP site was the most likely candidate gene for $n p s$. 


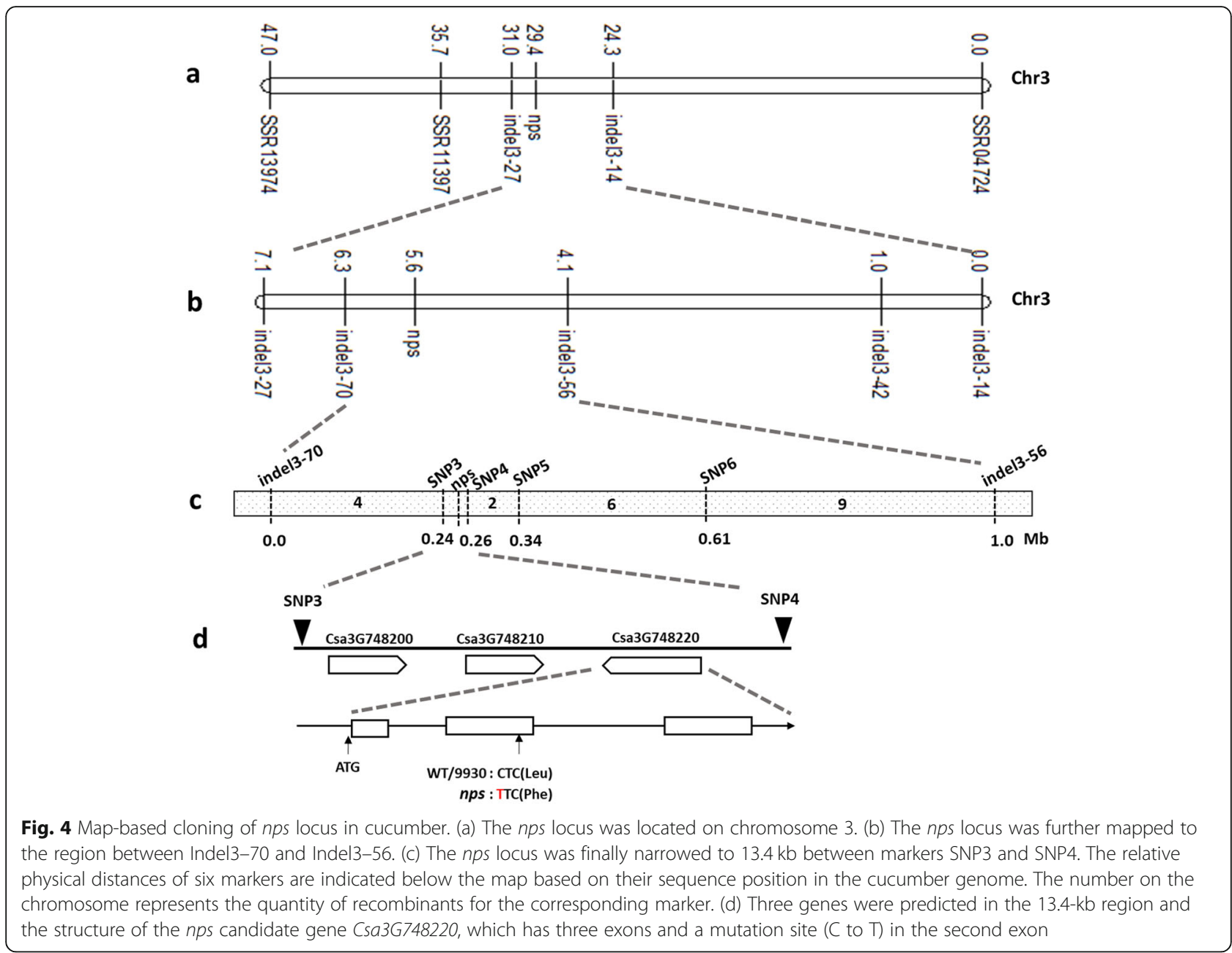

The genomic sequence of Csa3G748220 from 9930 and nps was cloned, and the full length of Csa3G748220 was $2930 \mathrm{bp}$ in the 9930 reference genome. Sequence alignment between 9930 and nps indicated that a single nucleotide mutation $(\mathrm{C} \rightarrow \mathrm{T})$ occurred in the second exon of the Csa3G748220 gene, which resulted in an amino acid change from Leucine to Phenylalanine. A previous study confirmed that the recessive mutant mict had a 2649-bp genomic deletion in Csa3G748220 and appeared to be glabrous with no noticeable trichomes on leaves, stems, branches and flowers, or spines on fruit [26]. Allelism test was also performed. Crossing nps and mict mutants, the $\mathrm{F}_{1}$ plants exhibited the microtrichome phenotype of mict. Among 132 nps $\times$ mict $\mathrm{F}_{2}$ individuals, 33 and 99 plants exhibited no pyramidshaped head trichomes and micro-trichome phenotypes, respectively, conforming to the 1:3 segregation ratio. These results indicated that the mutation sites of the two mutants occurred in the same gene (Figure S2), and the nps is an allelic mutation of mict, renamed as mictL130F.

\section{Transcriptome profile analysis}

To investigate the gene regulatory networks of Mict, comparative transcriptomic analysis of cucumber apical leaves was performed between 06 and 2 (mict) compared with its wild type 06-1 and nps (mict-L130F) compared with its wild type WD1. The transcriptomic data of 06-

Table 1 Predicted genes in the 13.4-kb genomic region of cucumber $\mathrm{Chr} 3$

\begin{tabular}{llll}
\hline Gene ID & BLASTX plant proteins & E-value & Annotation \\
\hline Csa3G748200 & AT2G36640 & $3.1 \mathrm{e}-75$ & Embryonic cell protein 63 \\
Csa3G748210 & AT2G36620 & $7.1 \mathrm{e}-76$ & 60 S ribosomal protein L24 \\
Csa3G748220 & AT5G03790 & $9.8 \mathrm{e}-42$ & Homeobox-leucine zipper protein \\
\hline
\end{tabular}


2 and $06-1$ is from previous study [40] and in this study the transcriptome of nps (mict-L130F) and WD1 was measured. We generated 49.46-51.38 million raw reads from each library, and 45.69-47.62 million clean reads were obtained after the removal of low-quality reads and adapter sequences. Among the clean reads, 93.9696.24\% were mapped to the gene database. Gene expression levels were calculated by FPKM values and differential expression was defined by statistical parameters $(P<0.05$ and fold change $>2$ or $<-2)$.

At a false discovery rate (FDR) of 0.05 , the group of mict-L130F vs WD1 was analyzed based on the RNASeq data of three independent biological replicates. A total of 500 differentially expressed genes (DEGs) were identified in mict-L130F vs WD1, including 194 upregulated and 306 down-regulated genes (Table S2). Further analysis found that 53 genes and 50 genes were upregulated or down-regulated respectively, in both groups, mict-L130F vs WD1 and 06-2 vs 06-1 (Fig. 5a, b). Therefore, the 103 DEGs were thought to be related to trichome development in cucumber. To determine the functions of these DEGs, gene ontology (GO) term enrichment and KEGG analysis were performed. The most significantly enriched GO terms were 'oxidationreduction process' in biological process, 'integral component of membrane' in cellular component and 'iron ion binding' in molecular function (Fig. 5c). Based on the KEGG database, pathway enrichment analysis was performed to identify significantly enriched plant hormone signal transduction, Glutathione metabolism, phenylpropanoid biosynthesis, Cyanoamino acid metabolism and MAPK signaling pathways (Fig. 5d).

Furthermore, we categorized the putative functions of these DEGs and focused on six genes which were involved in plant hormone pathway or encode critical transcription factors that can be linked to trichome formation based on work in other systems (Table 2). To further confirm the DEGs identified by RNA-Seq analysis, we tested the transcriptomic data by checking the expression of the genes shown in Table 2 with quantitative reverse transcription polymerase chain reaction (qRT-PCR) and found a good agreement with the transcriptome data (Figure S3). Except the JAZ1-like gene and IAA16-like gene, the relative expression fold change of mict-L130F/WD1 was higher than that of 06-2/06-1 in other genes (Fig. 5e). In Table 2, three genes encoding critical transcription factors can be linked to trichome development. The putative homolog of Csa1G051590 in Arabidopsis thaliana is an SPL9-like gene that promotes trichome formation in the adult phase by regulating miR172 and its target of Eat 1 (TOE1) and TOE2 [40, 41]. The putative homolog of Csa5G604260 in Arabidopsis thaliana encodes a HD-Zip I transcriptional activator involved in leaf and hypocotyl development. Its promoter is bound by PIF1 which likely regulates its expression [42]; The putative homolog of Csa1G555600

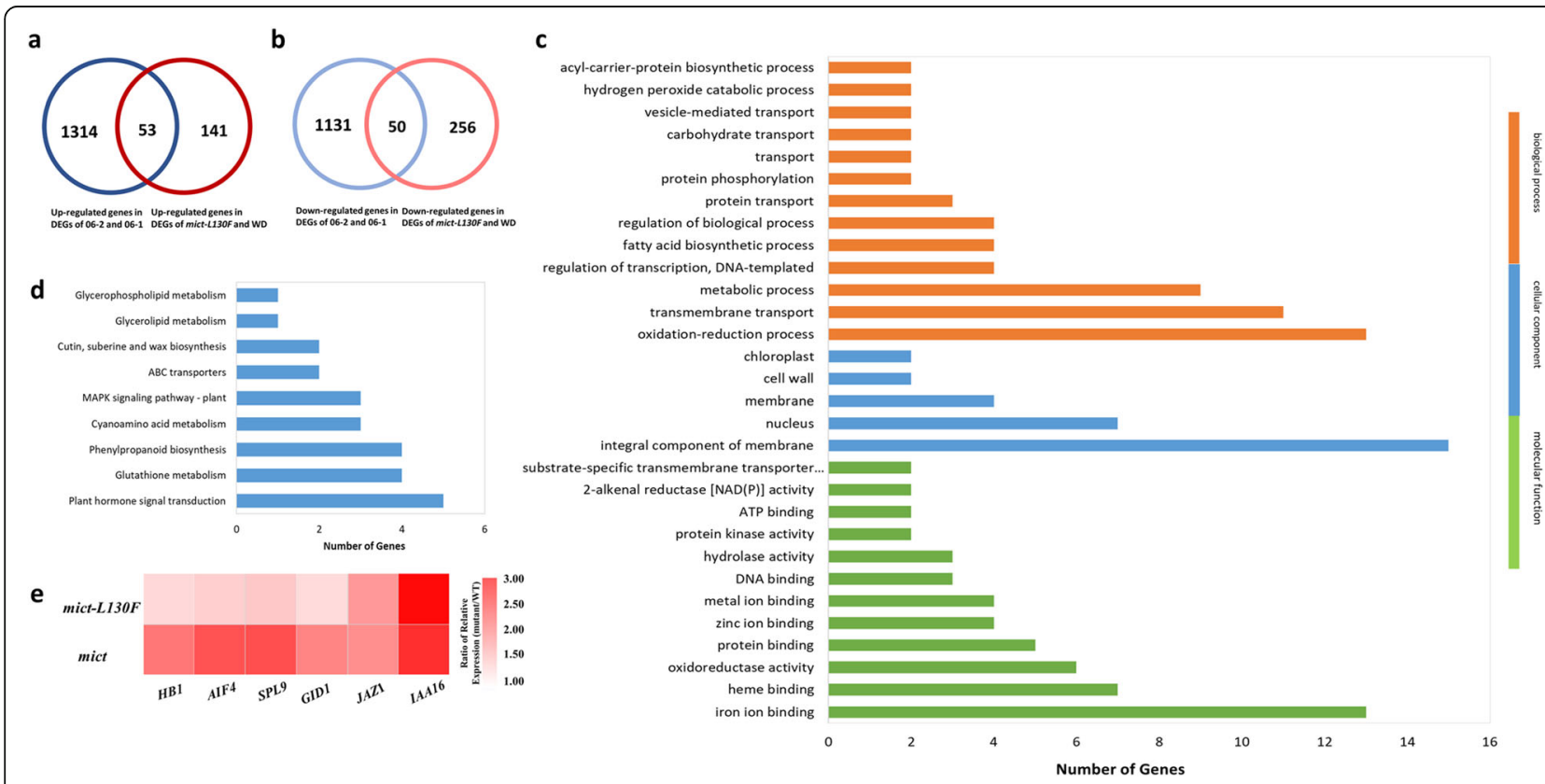

Fig. 5 Analysis of different expression genes between mict-L130F VS WD1 and 06-2 vs 06-1. (a) Venn diagrams of up-regulated genes in DEGs of mict-L130F VS WD1 (red) and 06-2 VS 06-1(blue) (b) Venn diagrams of down-regulated genes in DEGs of mict-L130F VS WD1 (red) and 06-2 VS 06-1(blue) (c) Go terms were categorized into biological process, cellular components and molecular functions based on the number of genes. (d) KEGG pathways that were enriched in the differentially expressed genes. (e) Expression of the candidate genes involved in the trichome development 
Table 2 Candidate genes involved in trichome formation of cucumber

\begin{tabular}{lll}
\hline Gene ID & Arabidopsis homolog & Annotation \\
\hline Csa1G051590 & AT2G42200.1 & Squamosa promoter binding protein SPL9-like \\
Csa5G604260 & AT3G01470.1 & Homeobox-leucine zipper-like protein HB1-like \\
Csa1G555600 & AT1G09250.1 & Transcription factor AIF4-like \\
Csa1G397130 & AT3G04730.1 & Auxin-responsive protein IAA16-like \\
Csa7G391240 & AT3G63010.1 & Gibberellin receptor GID1-like \\
Csa1G597690 & AT1G19180.1 & Jasmonate-zim-domain protein 1 JAZ1-like \\
\hline
\end{tabular}

in Arabidopsis thaliana negatively regulates cell elongation through a triantagonistic bHLH system [43].

In case to find out genes function on the different trichomes morphology, especially trichome elongation, we performed transcriptome analysis of $06-2$ vs mictL130F. A total of 2184 DEGs was identified. Among them, 1706 genes were down-regulated and 478 genes were up-regulated in mict-L130F. Expression trend analysis of 2184 DEGs was performed and nearly $81.3 \%$ DEGs were classified into 2 trends (Fig. 6a). SEM shows that the leaf trichomes length of WT and mict-L130F was comparatively similar and that of mict was significantly shorter. Trend 2 is similar to the trend of trichome length and Trend 1 is opposite, which means these genes may function on the length of trichomes. Phytohormone treatments influence cucumber trichome development and morphogenesis [44-46]. STRING analysis [47] was used to predict the functional association networks of the homologs to Arabidopsis among these DEGs. In the predicted protein interaction network, functionally related genes that were closely associated with auxin response were identified (Fig. 6b), which provide targets for further studies of trichome development.

\section{Discussion}

Cucumber fruits are economically valuable and fruit spines directly affect the appearance and perceived

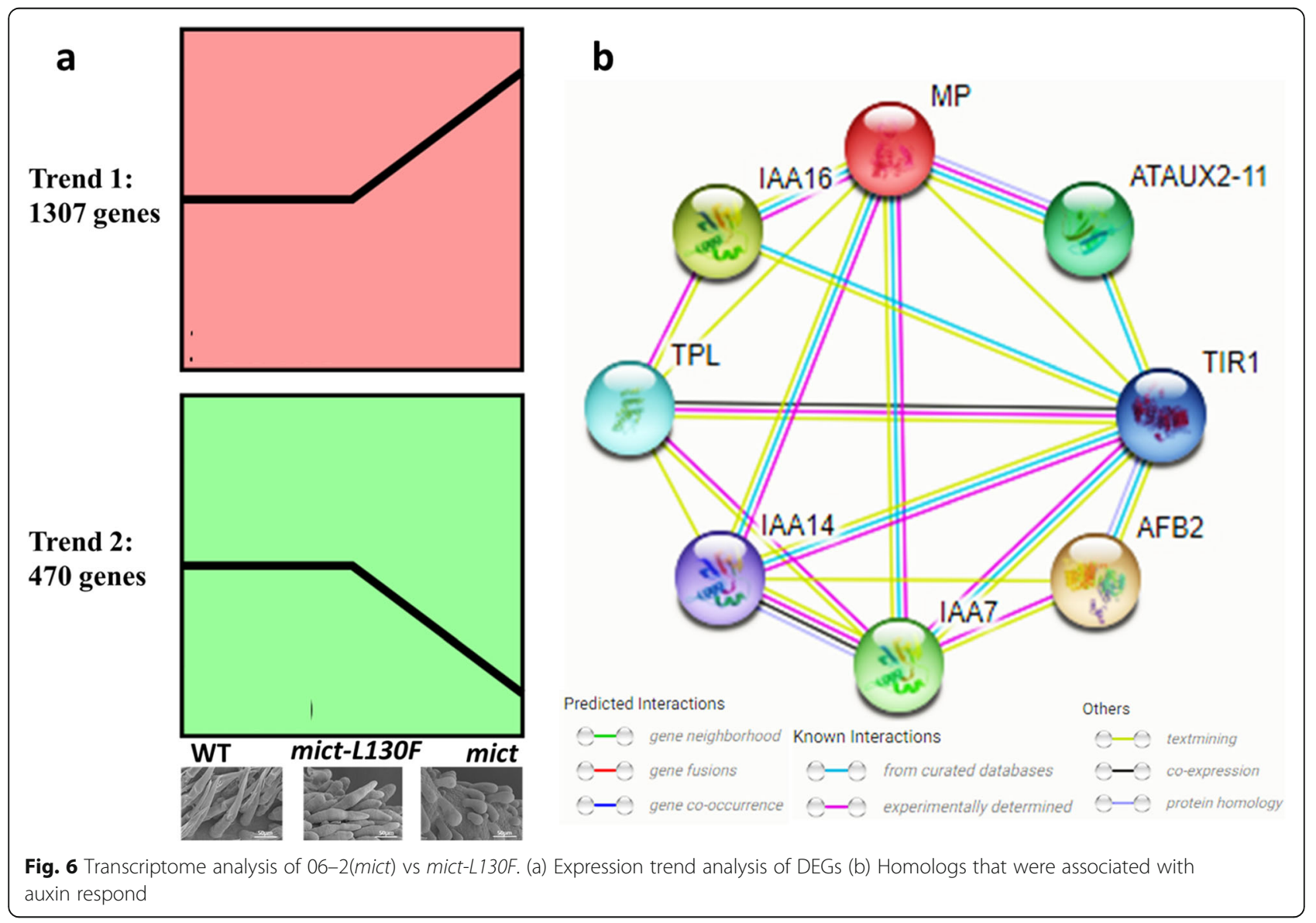


quality of the fruits. No pyramid-shaped head mutant (nps) used in this article was selected from the $\mathrm{M}_{2}$ generation of the WD1 mutant library, which was caused by EMS mutagenesis. Genetic studies indicated that the no pyramid-shaped head trait is recessive to the pyramidshaped head trait and the mict-L130F mutant in Mict gene induced a change in amino acid from leucine $(\mathrm{L})$ to phenylalanine $(F)$. In a previous study, the determination and morphogenesis of the multicellular trichomes of $\mathrm{cu}$ cumber were regulated by HD-ZIP transcription factors. The genes related to cucumber trichome (spine) development are tril, mict and $T u[20,26,27]$. In this study, mict-L130F showed an intermediate trichome phenotype between mict and wild type. The abnormal trichomes/ spines are all without pyramid-shaped heads and appear to be softer than those of the wild type, which is different from known types of trichomes.

The development of glandular trichomes has multiple stages, including initiation and expansion of the trichome precursor cell protuberating out of the epidermal surface, followed by periclinal bipartition to two cells (top and bottom) which later form the head region and the stalk, respectively, through subsequent cell divisions [45]. Disruption of the Mict locus led to abnormal trichomes with a greatly reduced number of cells, aberrant cell shapes and organization, and branched trichomes (Fig. 1), suggesting that the Mict gene may be required for cell division and directional growth of cucumber trichomes. By observing the mict-L130F and WT leaves continuously, we found that the formation of apical cells was prevented in stage III, but the elongation of trichomes was not affected in stage IV and stage $\mathrm{V}$ in mict-L130F. In previous study, we observed mict leaves in the same method and found that trichomes stayed in the bulge shape (stage III) without cell division or elongation [48]. In addition, the density of trichomes/spines in mict or mict-L130F are also higher than those of WT (Fig. 2, [26] These results implied that Mict functioned at the beginning of trichome development, particularly in trichome initiation from single-cell bulges to coneshaped multicellular trichomes.

According to allele verification, $F_{1}$ progeny are microtrichomes and $F_{2}$ progeny shows a ratio of 3:1 which confirm that mict and mict-L130F mutants are caused by allelic mutations of the same gene Csa3G748220. Different with mict-L130F mutants, in $\mathrm{F}_{1}$ progeny, there only single copy of mict-L130F and the expression level of the gene may be affected. It has been reported that Tril has a gene dosage effect on fruit spine density [35]. Thus, we speculated that whether there is also a gene dosage effect existing in Mict and further research are needed.

Based on our previous study, mict mutant leaves and fruit surfaces looked brighter and glossier and it has been confirmed that Mict can activate four genes (CsFLS1, CsTT4, CsCER26 and CsMYB36) involved in flavonoid and cuticular lipids biosynthesis which related to the appearance [48]. In this study, we also found that mict-L130F shows the similar phenotype with mict in wax and glossy (Fig. 1). Transcriptome profiling reveals that the four genes are all down-regulated both in mict and mict-L130F, and the expression levels of these genes were more affected in mict mutants. Consistent with Mict, in yeast one-hybrid assay, Mict-L130F can also activate these four genes (Fig. S4). It declares that the single amino acid mutant of Mict may also affect the regulation of secondary metabolites.

Plant trichome initiation and morphogenesis are influenced by diverse developmental and environmental cues [49-51]. KEGG analysis of these DEGs revealed that plant hormone signal transduction was affected in MictL130F and mict (Fig. 5d). The DEGs involved in the hormone pathway may be related to trichome formation, as listed in Table 2, and they were all up-regulated in both mict and Mict-L130F (Fig. 5e). Csa1G397130 is an AUX/ IAA transcriptional regulator family protein. Aux/IAAs proteins and auxin response factors (ARFs) are involved in auxin-dependent transcriptional regulation, and ARFs can act as either transcriptional activators or repressors of auxin-responsive genes [52, 53]. Down-regulation of SIIAA15 in tomato obviously decreased the formation of different types of trichomes, which indicates that auxindependent transcriptional regulation is involved in trichome initiation in tomato $[54,55]$. The putative homolog of Csa7G391240 in Arabidopsis thaliana encodes the gibberellin receptor. Gibberellin (GA) hormones are involved in many growth and developmental processes in plants. GA-deficient mutants, such as ga1-3, have almost completely glabrous leaves. The application of exogenous GAs to ga1-3 plants induces trichome formation [56]. Gibberellins promote trichome production in Arabidopsis through up-regulation of GL1 and TTG genes [57]. In cucumber, the application of GA showed a positive effect on fruit trichome initiation [45]. CsTTG1 acts in parallel to Mict/csgl1, a key trichome formation factor that regulates the initiation of fruit trichome and CsTTG1 can directly interact with Mict [58]. Whether the functions of GA on fruit spines are related to GL1 and TTG remains to be further studied. Csa1G597690 is a jasmonate-zim-domain (JAZ-like) protein that acts as a transcriptional repressor in the jasmonate (JA) hormonal response. The hormonal signals GA and JA antagonistically and synergistically regulate diverse aspects of plant growth, development, and defense. JA activates the WD-repeat/bHLH/MYB complex and induces initiation of trichomes in Arabidopsis [59]. In cucumber, trichomes are multicellular, unbranched cells that are regularly distributed on most 
aerial surfaces. The tril/csgl3 and csgll/mict are important cucumber mutants that enable the dissection of trichome development into distinct, genetically controlled steps, as follows: Tril regulates the initiation and density of the trichomes and Mict allows trichomes to differentiate correctly $[20,26]$. Both Tril and Mict are HD-ZIP transcription factors, different from the wellknown MYB-WD40-bHLH complex in Arabidopsis. The regulatory network for the formation of trichomes remains unknown. However, Tril and Mict may also be involved in the JA pathway and need more experimental data.

\section{Conclusions}

Through morphological observation, we found that several types of trichomes mutant forms existed in the no pyramid-shaped head trichomes mutant. Map-based cloning confirmed that the mutant was caused by the single amino acid substitution of the gene Csa3G748220, which is an allelic mutant of Mict. According to the transcriptome analysis of mict-L130F and mict, we identified that several genes may be related to the phenotype differences. Our results can help further facilitate understanding of the biological and molecular mechanisms of trichomes development.

\section{Abbreviations}

NPS: No pyramid-shaped head trichomes; EMS: Ethyl methane sulfonate; BSA: Bulked segregation analysis; InDel: Insertion deletion; SNP: Single nucleotide polymorphism; SSR: Simple Sequence Repeats; WT: Wild type; HD ZIP: Homeodomain-leucine zipper; dCAPS: derived cleaved amplified polymorphic sequences.; SEM: Scanning electron microscope; KEGG: Kyoto Encyclopedia of Genes and Genomes; GO: Gene Ontology; DEGs: Different Expression Genes

\section{Supplementary Information}

The online version contains supplementary material available at https://doi. org/10.1186/s12870-021-02955-1.

Additional file 1 Figure S1 Partical results of linkage analysis with dcaps-M demonstrating that this marker is co-segregated with the phenotype.

Additional file 2 Figure S2 Phenotype of leaves on nps, mict and $\mathrm{F}_{1}$ (nps $\times$ mict)

Additional file $\mathbf{3}$ Figure S3 $q R T-P C R$ confirmation of differentially expressed genes identified by transcriptome analysis.

Additional file 4 Figure S4 Mict-L130F activates the expression of CSTT4, CSFLS1, CSCER26, and CSMYB36.

Additional file 5 File S1 Amino acid sequence alignment of Mict between 10 cucumber natural lines and mutant $n p s$.

Additional file $\mathbf{6}$ Table $\mathbf{S} \mathbf{1}$ List of primers used in this study.

Additional file $\mathbf{7}$ Table $\mathbf{S 2}$ List of genes that are differentially expressed in the wild-type and mict-L130F mutant.

Additional file $\mathbf{8}$ Table $\mathbf{S 3}$ List of genes that are consistently differentially expressed in both groups, mict-L130F vs WD1 and 06-2 vs 06-1.

Additional file $\mathbf{9}$ Table S4 List of genes that are consistently differentially expressed in 06-2 vs mict-L130F.

\section{Acknowledgements}

We thank Hui-Ming Chen for giving cucumber lines 06-1 and 06-2. We thank the reviewers for critically reading the manuscript.

\section{Authors' contributions}

JS.P, G.W., D.L., and L.Z. conceived the project and designed the research. L.Z. performed most of the experiments with help from H.W., Y.C., H.D., J.P., K.Z. and H.H. L.Z. analyzed the data and wrote the article with assistance from R.C., G.W., and JS.P. L.Z. supervised the project and completed the writing. All authors have read and approved the manuscript.

\section{Funding}

This work was supported by the National Key R\&D Program of China (Grant No. 2018YFD0100701), the National Natural Science Foundation of China (31471156), Natural Science Foundation of Shanghai (20ZR1439600) and the Project of Science and Technology Commission of Shanghai Municipality (18391900300).

\section{Availability of data and materials}

All data generated or analyzed during this study are included in this published article [and its additional files].

\section{Declarations}

Ethics approval and consent to participate

Not applicable.

\section{Consent for publication}

Not applicable.

\section{Competing interests}

The authors declare that they have no competing financial interests.

\section{Author details}

${ }^{1}$ School of Agriculture and Biology, Shanghai Jiao Tong University, Shanghai 200240, China. ${ }^{2}$ State Key Laboratory of Vegetable Germplasm Innovation, Tianjin 300384, China.

Received: 21 January 2021 Accepted: 24 March 2021

Published online: 16 April 2021

References

1. Mauricio R, Rausher MD. Experimental manipulation of putative selective agents provides evidence for the role of natural enemies in the EVOLUTION of plant defense. Evolution. 1997;51(5):1435-44. https://doi.org/10.1111/j.1 558-5646.1997.tb01467.x.

2. Wagner GJ, Wang E, Shepherd RW. New approaches for studying and exploiting an old protuberance, the plant trichome. Ann Bot. 2004;93(1):311. https://doi.org/10.1093/aob/mch011.

3. Serna L, Martin C. Trichomes: different regulatory networks lead to convergent structures. Trends Plant Sci. 2006;11(6):274-80. https://doi.org/1 0.1016/j.tplants.2006.04.008.

4. Schnittger A, Folkers U, Schwab B, Jürgens G, Hülskamp M. Generation of a spacing pattern: the role of triptychon in trichome patterning in Arabidopsis. Plant Cell. 1999;11(6):1105-16. https://doi.org/10.1105/tpc.11. 6.1105.

5. Kennedy GG. Tomato, pests, parasitoids, and predators: tritrophic interactions involving the genus Lycopersicon. Annu Rev Entomol. 2003; 48(1):51-72. https://doi.org/10.1146/annurev.ento.48.091801.112733.

6. Balkunde R, Pesch M, Hülskamp M. Trichome patterning in Arabidopsis thaliana from genetic to molecular models. Curr Top Dev Biol. 2010;91:299321. https://doi.org/10.1016/S0070-2153(10)91010-7.

7. Oppenheimer DG, Herman PL, Sivakumaran S, Esch J, Marks MD. A myb gene required for leaf trichome differentiation in Arabidopsis is expressed in stipules. Cell. 1991;67(3):483-93. https://doi.org/10.1016/ 0092-8674(91)90523-2.

8. Rerie WG, Feldmann KA, Marks MD. The GLABRA2 gene encodes a homeo domain protein required for normal trichome development in Arabidopsis. Genes Dev. 1994;8(12):1388-99. https://doi.org/10.1101/gad.8.12.1388.

9. Walker AR, Davison PA, Bolognesi-Winfield AC, James CM, Srinivasan N, Blundell TL, et al. The TRANSPARENT TESTA GLABRA1 locus, which regulates 
trichome differentiation and anthocyanin biosynthesis in Arabidopsis, encodes a WD40 repeat protein. Plant Cell. 1999;11(7):1337-50. https://doi. org/10.1105/tpc.11.7.1337.

10. Payne $C T$, Zhang F, Lloyd AM. GL3 encodes a bHLH protein that regulates trichome development in arabidopsis through interaction with GL1 and TTG1. Genetics. 2000;156(3):1349-62.

11. Bernhardt C, Lee MM, Gonzalez A, Zhang F, Lloyd A, Schiefelbein J. The bHLH genes GLABRA3 (GL3) and ENHANCER OF GLABRA3 (EGL3) specify epidermal cell fate in the Arabidopsis root. Development. 2003;130(26): 6431-9. https://doi.org/10.1242/dev.00880.

12. Zhao M, Morohashi K, Hatlestad G, Grotewold E, Lloyd A. The TTG1-bHLHMYB complex controls trichome cell fate and patterning through direct targeting of regulatory loci. Development. 2008;135(11):1991-9. https://doi. org/10.1242/dev.016873.

13. Grebe M. The patterning of epidermal hairs in Arabidopsis--updated. Curr Opin Plant Biol. 2012;15(1):31-7. https://doi.org/10.1016/j.pbi.2011.10.010.

14. Marks MD, Wenger JP, Gilding E, Jilk R, Dixon RA. Transcriptome analysis of Arabidopsis wild-type and gl3-sst Sim trichomes identifies four additional genes required for trichome development. Mol Plant. 2009;2(4):803-22. https://doi.org/10.1093/mp/ssp037.

15. Wada T, Tachibana T, Shimura Y, Okada K. Epidermal cell differentiation in Arabidopsis determined by a Myb homolog. CPC Science. 1997;277(5329): 1113-6. https://doi.org/10.1126/science.277.5329.1113.

16. Kirik V, Simon M, Huelskamp M, Schiefelbein J. The ENHANCER OF TRY AND CPC1 gene acts redundantly with TRIPTYCHON and CAPRICE in trichome and root hair cell patterning in Arabidopsis. Dev Biol. 2004;268(2):506-13. https://doi.org/10.1016/j.ydbio.2003.12.037.

17. Wang S, Kwak SH and Zeng Qm, Ellis BE, Chen XY, Schiefelbein J, Chen JG TRICHOMELESS1 regulates trichome patterning by suppressing GLABRA1 in Arabidopsis. Development. 2007; 134: 3873-3882, 21, DOl: https://doi.org/1 0.1242/dev.009597.

18. Wester K, Digiuni S, Geier F, Timmer J, Fleck C, Hulskamp M. Functional diversity of R3 single-repeat genes in trichome development. Development. 2009;136(9):1487-96. https://doi.org/10.1242/dev.021733.

19. Wang S, Hubbard L, Chang Y, Guo J, Schiefelbein J, Chen JG. Comprehensive analysis of single-repeat R3 MYB proteins in epidermal cell patterning and their transcriptional regulation in Arabidopsis. BMC Plant Biol. 2008:8(1):81. https://doi.org/10.1186/1471-2229-8-81.

20. Wang $Y$, Nie J, Chen $H$, et al. Identification and mapping of Tril, a homeodomain-leucine zipper gene involved in multicellular trichome initiation in Cucumis sativus. Theor Appl Genet. 2016;129(2):305-16. https:// doi.org/10.1007/s00122-015-2628-4.

21. Pan Y, Bo K, Cheng Z, Weng Y. The loss-of-function GLABROUS 3 mutation in cucumber is due to LTR-retrotransposon insertion in a class IV HD-ZIP transcription factor gene CsGL3 that is epistatic over CsGL1. BMC Plant Biol. 2015;15(1):302. https://doi.org/10.1186/s12870-015-0693-0.

22. Cui J, Miao H, Ding L, et al. A New Glabrous Gene (csgl3) Identified in Trichome Development in Cucumber (Cucumis sativus L.). Plos One. 2016;11: e148422.

23. Zhang $H$, Wang $L$, Zheng $S$, et al. A fragment substitution in the promoter of CsHDZIV11/CsGL3 is responsible for fruit spine density in cucumber (Cucumis sativus L.). Theor Appl Genet. 2016;129:1289-301.

24. Chen C, Liu M, Jiang L, Liu X, Zhao J, Yan S, et al. Transcriptome profiling reveals roles of meristem regulators and polarity genes during fruit trichome development in cucumber (Cucumis sativus L.). J Exp Bot. 2014; 65(17):4943-58. https://doi.org/10.1093/jxb/eru258.

25. Li Q, Cao C, Zhang C, Zheng S, Wang Z, Wang L, et al. The identification of Cucumis sativus Glabrous 1 (CsGL1) required for the formation of trichomes uncovers a novel function for the homeodomain-leucine zipper I gene. J Exp Bot. 2015;66(9):2515-26. https://doi.org/10.1093/jxb/erv046.

26. Zhao JL, Pan JS, Guan Y, Zhang WW, Bie BB, Wang YL, et al. Micro-trichome as a class I homeodomain-leucine zipper gene regulates multicellular trichome development in Cucumis sativus. J Integr Plant Biol. 2015;57(11): 925-35. https://doi.org/10.1111/jipb.12345.

27. Yang $X$, Zhang $W$, He $H$, et al. Tuberculate fruit gene Tu encodes a $\mathrm{C}_{2} \mathrm{H} 2$ zinc finger protein that is required for the warty fruit phenotype in cucumber (Cucumis sativus L.). Plant J. 2014;78:1034-46.

28. Shi $P$, Fu X, Shen Q, Liu M, Pan Q, Tang Y, et al. The roles of AaMIXTA1 in regulating the initiation of glandular trichomes and cuticle biosynthesis in Artemisia annua. New Phytologist. 2018;217(1):261-76. https://doi.org/1 $0.1111 /$ nph.14789.
29. Michelmore RW, Paran I, Kesseli RV. Identification of markers linked to disease-resistance genes by bulked segregant analysis: a rapid method to detect markers in specific genomic regions by using segregating populations. Proc Natl Acad Sci U S A. 1991;88(21):9828-32. https://doi.org/1 0.1073/pnas.88.21.9828.

30. Cavagnaro PF, Senalik DA, Yang L, Simon PW, Harkins TT, Kodira CD, et al. Genome-wide characterization of simple sequence repeats in cucumber (Cucumis sativus L.). BMC Genomics. 2010;11(1):569. https://doi.org/10.11 86/1471-2164-11-569.

31. Yang L, Koo DH and Li Ym, Zhang X, Luan F, Havey MJ, Jiang J, Weng Y Chromosome rearrangements during domestication of cucumber as revealed by high-density genetic mapping and draft genome assembly. Plant J 2012; 71: 895-906, 6, DOI: https://doi.org/10.1111/j.1365-313X.2012. 05017.x.

32. Zhang WW, Pan JS, He HL, et al. Construction of a high density integrated genetic map for cucumber (Cucumis sativus L.). Theor Appl Genet. 2012;124: 249-59.

33. Guo C, Yang X, Wang Y, Nie J, Yang Y, Sun J, et al. Identification and mapping of ts (tender spines), a gene involved in soft spine development in Cucumis sativus. Theor Appl Genet. 2018;131(1):1-12. https://doi.org/10.1 007/s00122-017-2954-9.

34. Sun J, Xiao T, Nie J, Chen Y, Lv D, Pan M, et al. Mapping and identification of CsUp, a gene encoding an Auxilin-like protein, as a putative candidate gene for the upward-pedicel mutation (up) in cucumber. BMC Plant Biol. 2019;19(1):157. https://doi.org/10.1186/s12870-019-1772-4.

35. Du H, Wang G, Pan J, et al. The HD-ZIP IV transcription factor Tril regulates fruit spine density through gene dosage effects in cucumber. J Exp Bot. 2020;71(20):6297-310. https://doi.org/10.1093/jxb/eraa344.

36. Fehlmann T, Reinheimer S, Geng C, Su X, Drmanac S, Alexeev A, et al. cPASbased sequencing on the BGISEQ-500 to explore small non-coding RNAs. Clin Epigenetics. 2016;8(1):123. https://doi.org/10.1186/s13148-016-0287-1.

37. Langmead B, Trapnell C, Pop M, Salzberg SL. Ultrafast and memory-efficient alignment of short DNA sequences to the human genome. Genome Biol. 2009;10(3):R25. https://doi.org/10.1186/gb-2009-10-3-r25.

38. Huang S, Li R, Zhang Z, Li L, Gu X, Fan W, et al. The genome of the cucumber, Cucumis sativus L. Nat Genet. 2009;41(12):1275-81. https://doi. org/10.1038/ng.475.

39. Tarazona S, García-Alcalde F, Dopazo J, et al. Differential expression in RNAseq: a matter of depth. Genome Res. 2011;21(12):2213-23. https://doi.org/1 $0.1101 /$ gr.124321.111.

40. Pan J, Zhang L, Chen G, et al. Study of micro-trichome (mict) reveals novel connections between transcriptional regulation of multicellular trichome development and specific metabolism in cucumber. Hortic Res-England. 2021;8(1):21.

41. Wu G, Park MY, Conway SR, Wang JW, Weigel D, Poethig RS. The sequential action of miR156 and miR172 regulates developmental timing in Arabidopsis. Cell. 2009;138(4):750-9. https://doi.org/10.1016/j.cell.2009.06.031.

42. Capella M, Ribone PA, Arce AL, Chan RL. Arabidopsis thaliana HomeoBox 1 (AtHB1), a Homedomain-leucine zipper I (HD-zip I) transcription factor, is regulated by PHYTOCHROME-INTERACTING FACTOR 1 to promote hypocotyl elongation. New Phytologist. 2015;207(3):669-82. https://doi.org/1 $0.1111 /$ nph.13401

43. Ikeda M, Mitsuda N, Ohme-Takagi M. ATBS1 INTERACTING FACTORs negatively regulate Arabidopsis cell elongation in the triantagonistic bHLH system. Plant Signal Behav. 2013;8(3):e23448. https://doi.org/10.4161/ psb.23448.

44. Zhang Y, Shen J, Bartholomew ES, Dong M, Chen S, Yin S, et al. TINY BRANCHED HAIR functions in multicellular Trichome development through an ethylene pathway in Cucumis sativus L. Plant J. 2021. https://doi.org/1 0.1111/tpj.15198.

45. Xue S, Dong M, Liu X, Xu S, Pang J, Zhang W, et al. Classification of fruit trichomes in cucumber and effects of plant hormones on type II fruit trichome development. Planta. 2019;249(2):407-16. https://doi.org/10.1007/ s00425-018-3004-9.

46. Liu X, Wang T, Bartholomew E, et al. Comprehensive analysis of NAC transcription factors and their expression during fruit spine development in cucumber (Cucumis sativus L.). Hortic Res-England. 2018;5:31.

47. Szklarczyk D, Franceschini A, Wyder S, Forslund K, Heller D, Huerta-Cepas J, et al. STRING v10: protein-protein interaction networks, integrated over the tree of life. Nucleic Acids Res. 2015;43(D1):D447-52. https://doi.org/10.1093/ nar/gku1003. 
48. Pan J, Zhang L, Chen G, Wen H, Chen Y, du H, et al. Study of microtrichome (mict) reveals novel connections between transcriptional regulation of multicellular trichome development and specific metabolism in cucumber. Hortic Res-England. 2021;8(1):21. https://doi.org/10.1038/s4143 8-020-00456-0.

49. Greenboim-Wainberg Y, Maymon I, Borochov R, Alvarez J, Olszewski N, Orì $\mathrm{N}$, et al. Cross talk between gibberellin and cytokinin: the Arabidopsis GA response inhibitor SPINDLY plays a positive role in cytokinin signaling. Plant Cell. 2005;17(1):92-102. https://doi.org/10.1105/tpc.104.028472.

50. Gan Y, Liu C, Yu H, Broun P. Integration of cytokinin and gibberellin signalling by Arabidopsis transcription factors GIS, ZFP8 and GIS2 in the regulation of epidermal cell fate. Development. 2007;134(11):2073-81. https://doi.org/10.1242/dev.005017.

51. Pattanaik S, Patra B, Singh SK, et al. An overview of the gene regulatory network controlling trichome development in the model plant. Arabidopsis Front Plant Sci. 2014;5:259.

52. Dharmasiri N, Estelle M. Auxin signaling and regulated protein degradation. Trends Plant Sci. 2004;9(6):302-8. https://doi.org/10.1016/j.tplants.2004.04 003.

53. Ren Z, Li Z, Miao Q, Yang Y, Deng W, Hao Y. The auxin receptor homologue in Solanum lycopersicum stimulates tomato fruit set and leaf morphogenesis. J Exp Bot. 2011;62(8):2815-26. https://doi.org/10.1093/jxb/ erq455.

54. Deng W, Yan F, Liu M, Wang X, Li Z. Down-regulation of SIIAA15 in tomato altered stem xylem development and production of volatile compounds in leaf exudates. Plant Signal Behav. 2012;7(8):911-3. https://doi.org/10.4161/ psb.20723.

55. Zhang X, Yan F, Tang Y, Yuan Y, Deng W, Li Z. Auxin response gene SIARF3 plays multiple roles in tomato development and is involved in the formation of epidermal cells and Trichomes. Plant Cell Physiol. 2015;56(11): 2110-24. https://doi.org/10.1093/pcp/pcv136.

56. Chien JC, SussX IM. Differential regulation of Trichome formation on the Adaxial and Abaxial leaf surfaces by gibberellins and photoperiod in Arabidopsis thaliana (L.) Heynh. Plant Physiol. 1996;111(4):1321-8. https://doi. org/10.1104/pp.111.4.1321.

57. Perazza D, Vachon G, Herzog M. Gibberellins promote Trichome formation by up-regulating GLABROUS1 in Arabidopsis1. Plant physiology (Bethesda). 1998;117(2):375-83. https://doi.org/10.1104/pp.117.2.375.

58. Chen C, Yin S, Liu X, et al. CsTTG1 encodes a WD-repeat protein that regulates fruit wart formation in Cucumis sativus through interaction with the Homeodomain-leucine zipper I protein Mict. Plant Physiol. 2016;171(2): 1156-1168.

59. Qi T, Huang H, Wu D, Yan J, Qi Y, Song S, et al. Arabidopsis DELLA and JAZ proteins bind the WD-repeat/bHLH/MYB complex to modulate gibberellin and Jasmonate signaling synergy. Plant Cell. 2014;26(3):1118-33. https://doi. org/10.1105/tpc.113.121731.

\section{Publisher's Note}

Springer Nature remains neutral with regard to jurisdictional claims in published maps and institutional affiliations.

Ready to submit your research? Choose BMC and benefit from:

- fast, convenient online submission

- thorough peer review by experienced researchers in your field

- rapid publication on acceptance

- support for research data, including large and complex data types

- gold Open Access which fosters wider collaboration and increased citations

- maximum visibility for your research: over $100 \mathrm{M}$ website views per year

At $\mathrm{BMC}$, research is always in progress.

Learn more biomedcentral.com/submissions 